\title{
Theoretical study on the molecular structure, intermolecular interaction and spectral features of 2-aminopyridine/ 2,3-dichloro-5,6-dicyano-1,4-benzoquinone complex
}

\author{
HAILONG WANG* \\ College of Biological, Chemical Sciences and Engineering, Jiaxing University, Jiaxing 314001, China \\ E-mail: wanghailong@mail.zjxu.edu.cn
}

MS received 17 January 2017; revised 13 March 2017; accepted 8 April 2017

\begin{abstract}
Density function theory (DFT) was performed to study the structures and binding energies of the 2-aminopyridine(2-AP)/2,3-dichloro-5,6-dicyano-1,4-benzoquinone (DDQ) complex. Four distinct stable conformers (denoted as S1-S4) were located and the bonding characteristics of these conformers were investigated with NBO and AIM analysis. It was revealed that $\mathbf{S 1}$ is formed by the edge(2-AP)-to-face(DDQ) linkage, which is stabilized by a moderate $\sigma-\pi$ interaction and hydrogen binding interaction. $\mathbf{S 2}$ and $\mathbf{S 3}$ are formed by the face(2-AP)-to-face(DDQ) linkage through $\sigma-\pi$ and $\pi-\pi$ interactions, and $\mathbf{S} \mathbf{4}$ is constructed by the edge(2-AP)-to-edge(DDQ) linkage through halogen bonding and hydrogen bonding interactions. The electronic excitation energies of the two stable conformers, $\mathbf{S 2}$ and $\mathbf{S 3}$, were calculated with time-dependent DFT (TDDFT), which revealed that the 2-AP $(\pi) \rightarrow \mathrm{DDQ}\left(\pi^{*}\right)$ charge transfer transitions in conformers $\mathbf{S} 2$ and S3 contribute to the two new charge transfer absorption bands which were experimentally observed at $598 \mathrm{~nm}$ and $557 \mathrm{~nm}$ for the 2-AP/DDQ complex in chloroform.
\end{abstract}

Keywords. Density functional theory; 2-AP/DDQ complex; NBO; AIM.

\section{Introduction}

Charge transfer (CT) complexes originating from intermolecular interactions between electron donors and accepters have been extensively investigated due to their various applications in organic electronics, optical devices and organic synthesis. ${ }^{1-3}$ A thorough understanding of the intermolecular interactions between the electron donor and accepter is of considerable fundamental and practical interest. Nitrogen-containing aromatic heterocyclic compounds can function as $n$ and $\pi$ type of electron donors to form charge transfer complexes with different electron accepters. ${ }^{4-9}$ Among them, aminopyridine (AP) compounds are of special interest because of their significant roles in both biological and chemical processes. 2-AP dimer can be considered as a mimetic model to study the lightinduced single and double proton transfer processes of Watson-Crick DNA base pairs. ${ }^{10}$ 2-AP/2-pyridone complex linked through antiparallel $\mathrm{N}-\mathrm{H} \cdots \mathrm{O}=\mathrm{C}$ and $\mathrm{N}-\mathrm{H} \cdots \mathrm{N}$ hydrogen bonds provided a model for the Watson-Crick hydrogen bonding configuration of the adenine-thymine and adenine-uracil nucleobase pairs. ${ }^{11}$ Moreover, AP as an analytical regent

\footnotetext{
*For correspondence
}

has many uses in analytical chemistry. It has been recognized that quinone compounds play a vital role in the biological electron transport processes such as photosystems I and II of photosynthesis and aerobic respiration. $^{12}$ 2,3-dichloro-5,6-dicyano-1,4-benzoquinone (DDQ) is a well-known member of the quinone family, and its charge transfer complexes formed with many electron donors present novel optical and electronic properties. ${ }^{13,14}$ In the past few years, several kinds of charge transfer complexes formed by AP donors and DDQ acceptors have been prepared and characterized by UV-Vis and infrared spectra. The stoichiometries and the formation constants of the studied complexes were determined from photometric titration methods. ${ }^{7-9}$

However, the basic issues of the AP/DDQ complex, such as the preferable geometry, the bonding characteristics, and the nature of the observed new absorption bands, have not been well understood. Therefore, in the present article, we report our experimental and theoretical results of the 2-AP/DDQ complex. The preferable geometries and binding energies of the complex were studied using density functional theory (DFT). Natural bond orbital analysis and topological analysis of electron densities were employed to explore the nature and the strength of the intermolecular interactions. In 
addition, the electronic excited states were calculated with time-dependent density function theory (TDDFT) to provide a deeper understanding of the observed UVVis absorption spectrum of the 2-AP/DDQ complex.

\section{Computational}

The molecular structures and binding energies of 2-AP/DDQ complex in the ground states were calculated with M06-2xD3 method, ${ }^{15}$ which has been applied to study a number of intermolecular interactions successfully. ${ }^{16-18}$ Vibrational frequency computations for the optimized structures were done to confirm the optimized structure to be an energy minimum and to evaluate the zero point energy (ZPE). The 6-31+ G(d) basis set was used in the structure optimizations and frequency calculations. Boys and Bernardi's counterpoise (CP) method was applied to correct the basis set superposition errors (BSSEs). ${ }^{19}$ In order to estimate the influence of the basis set on the binding energy, the interaction energies were calculated with larger basis sets at the optimized geometry.

Charge transfer from 2-AP to DDQ was estimated using natural population analysis (NPA). Natural bond orbital (NBO) analysis and Bader's atoms-in-molecule (AIM) analysis were performed to explore the nature and strength of intermolecular interactions between 2-AP and DDQ in the complex. ${ }^{20,21}$ The electronic excitation energies and oscillator strengths of the 2-AP/DDQ complex were calculated with time-dependent density functional theory (TDDFT) using PBE1PBE methods with 6-311G(d,p) basis set, and solvation effect (chloroform) was considered by using the integral equation formalism polarisable continuum model (IEFPCM). ${ }^{22,23}$ All DFT calculations were performed using Gaussian 09 program. ${ }^{24}$

\section{Results and Discussion}

\subsection{Structural geometry and binding energy}

The possible conformations of the 2-AP/DDQ complex was fully optimized in gas phase without any symmetry constraints using M06-2x-D3/6-31+G(d) method. The stoichiometry of 2-AP and DDQ is $1: 1$ which is adopted from the literature. ${ }^{9}$ Four distinct stable conformers, denoted as S1, S2, S3 and S4, were obtained and these are displayed in Figure 1. Vibrational frequencies were calculated based on the optimized geometries and absence of negative frequencies confirmed that the four conformers were local minimum-energy structures.

As shown in Figure 1, S1 has a slightly inclined Tshape structure with 2-AP perpendicular to the mean plane of DDQ ring. DDQ displays slightly nonlinear distortion with $\mathrm{O}_{8}$ away from 2-AP. The distance between $\mathrm{C}_{3}$ and $\mathrm{N}_{17}$ is $2.67 \AA$, which is much shorter than the sum of the van der Waals radii of $\mathrm{C}$ and $\mathrm{N}$ atoms (3.25 $\AA)^{25}$ and shows that it exists a moderately strong attractive interaction between the two atoms. In addition, the $\mathrm{O}_{8} \ldots \mathrm{H}_{26}$ distance $(2.15 \AA)$ is significantly shorter than the sum of the van der Waals radii of $\mathrm{O}$ and $\mathrm{H}$ atoms $(2.72 \AA),{ }^{25}$ which indicates a rather strong hydrogen bonding interaction between DDQ and 2-AP. For S2 and S3, the positions of 2-AP and DDQ present parallel-displaced orientations. The vertical and horizontal displacements are $3.02 \AA$ and $1.85 \AA$ for $\mathbf{S 2}$ and $3.03 \AA$ and $1.82 \AA$ for $\mathbf{S 3}$, respectively. These distances are compatible with the typical stacking interaction. The positions of 2-AP and DDQ of conformer $\mathbf{S 4}$ are nearly in the same plane. The distance $(2.93 \AA)$ between $\mathrm{N}_{17}$ and $\mathrm{Cl}_{10}$, which is slightly shorter than the sum of the van der Waals radii of $\mathrm{Cl}$ and $\mathrm{N}$ atoms $(3.30 \AA),{ }^{25}$ indicates that there is moderately strong halogen bonding between the two atoms. In addition, the distances of $\mathrm{O}_{8} \ldots \mathrm{H}_{26}$ and $\mathrm{Cl}_{10} \ldots \mathrm{H}_{26}$ are $2.47 \AA$ and $2.66 \AA$, respectively, which are nearly close to the sum of the van der Waals radii of $\mathrm{O}$ and $\mathrm{H}$ atoms $(2.72 \AA)$ and $\mathrm{Cl}$ and $\mathrm{H}$ atoms $(2.81 \AA),{ }^{25}$ and weak hydrogen bonding interactions between 2 -AP and DDQ seem to exist.

In order to estimate the influence of basis set on the binding energy, the binding energies of S1-S4 were calculated using 6-31++G(d,p) and 6-311G(d,p) basis sets at the M06-2X-D3/6-31+G(d) optimized geometries through counterpoise $(\mathrm{CP})$ and zero point energy (ZPE) corrections. The calculated results are tabulated in Table 1. It is shown that the binding energies of S1-S4 calculated with 6-31++G(d,p) and 6-311G(d,p) basis sets are compatible with those calculated with 6 $31+\mathrm{G}(\mathrm{d})$ basis set, which indicates that a moderate basis set, $6-31+\mathrm{G}(\mathrm{d})$, could be used in the calculation of the binding energy.

\subsection{NPA and NBO analysis}

Charge transfer from the donor (2-AP) to the accepter (DDQ) was estimated using NPA with M06-2X-D3/6$311 \mathrm{G}(\mathrm{d}, \mathrm{p})$ method. The results revealed that there is a positive charge on the 2-AP part of S1-S4 $(0.0718 \mathrm{e}$, $0.1117 \mathrm{e}, 0.1183 \mathrm{e}$, and $0.0080 \mathrm{e}$, respectively) whereas an equivalent negative charge is on the DDQ unit. The charge distributions indicate a net charge flow from the donor of 2-AP to the accepter of DDQ.

To better understand the intermolecular interaction of the 2-AP/DDQ complex, NBO analysis has been carried out with M06-2X-D3/6-311G(d,p) method, which provides much information about the nature and strength of intermolecular interactions from the viewpoint of local orbital interactions and their second order perturbation energy $\left(E^{(2)}\right) .{ }^{20}$ Table 2 lists the $E^{(2)}$ values of selected 


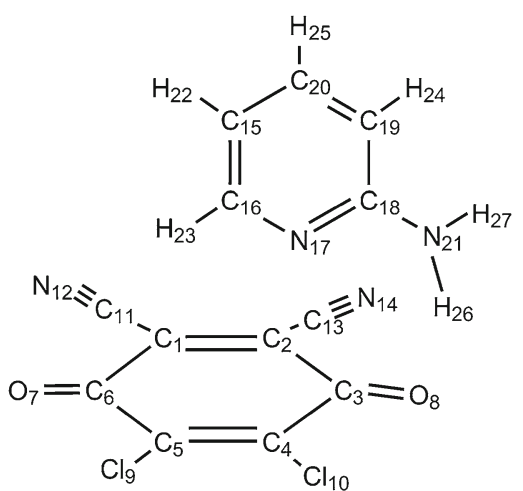

S1 (47.20)

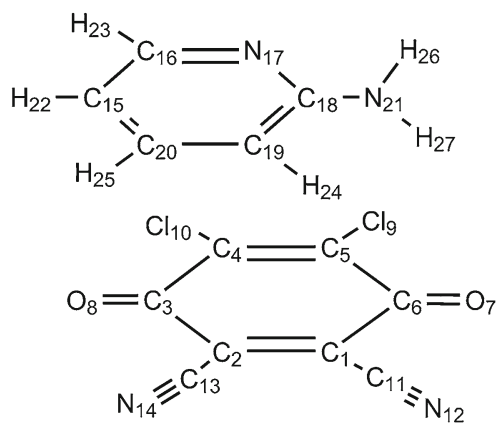

S3 (63.12)

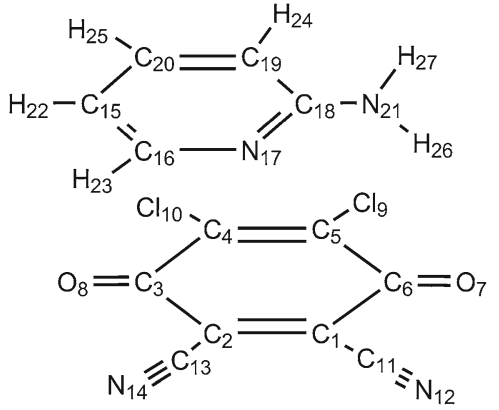

S2 (64.16)

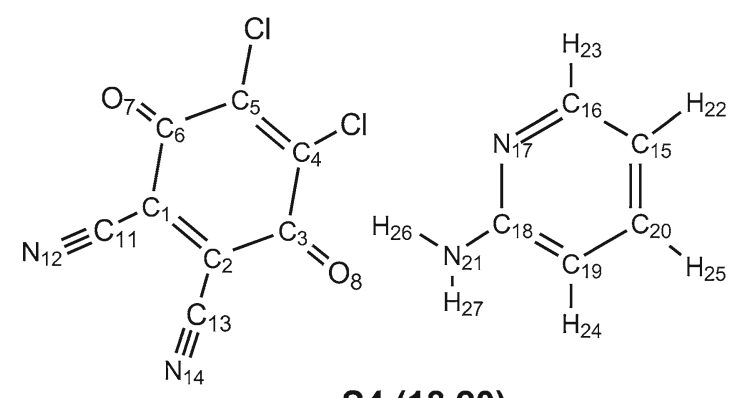

S4 (18.20)

Figure 1. Four possible orientations (denoted as S1-S4) and the labels of the atoms of the 2-AP/DDQ complex. The binding energies (in kcal/mol) of S1-S4 shown in the brackets were calculated with M06-2x-D3/6-31+G(d) method.

Table 1. The CP and ZPE-corrected binding energies (kJ/mol) of $\mathbf{S 1 - S 4}$ and their basis set dependence.

\begin{tabular}{lllll}
\hline (Method //Geometry) & S1 & S2 & S3 & S4 \\
\hline M06-2X-D3/6-31+G(d)//6-31+G(d) & 47.20 & 64.16 & 63.12 & 18.20 \\
M06-2X-D3/6-31++G(d,p) $^{\text {a } / / 6-31+G(d) ~}$ & 47.83 & 63.47 & 62.36 & 18.76 \\
M06-2X-D3/6-311G(d,p) $^{\text {a } / / 6-31+G(d) ~}$ & 48.21 & 64.22 & 65.85 & 19.11 \\
\hline
\end{tabular}

${ }^{a}$ ZPE correction from the M06-2X-D3/6-31 + G(d) result

interactions between the local orbitals where BD, LP, $\mathrm{BD}^{*}$ denote the occupied bonding orbitals, the lone pairs and the empty antibonding orbitals, respectively.

As shown in Table 2, NBO analysis of $\mathbf{S 1}$ indicates that the interaction between 2-AP and DDQ originates mainly from the migration of the $n$ lone pairs of $\mathrm{N}_{17}$ to the $\pi^{*}$ orbital of the $\mathrm{C}_{3}-\mathrm{O}_{8}$ carbonyl group and the $\mathrm{C}_{1}-\mathrm{C}_{2}$ double bond. The corresponding $\mathrm{E}^{(2)}$ value are 5.97 and $3.06 \mathrm{kcal} / \mathrm{mol}$, respectively. It is also noted that there exists a strong hydrogen bonding interaction between $\mathrm{O}_{8}$ and $\mathrm{H}_{26}$ which can be characterized by the orbital interaction $\mathrm{LP}(\sigma) \mathrm{O}_{8} \rightarrow \mathrm{BD}^{*}(\sigma) \mathrm{N}_{21}-\mathrm{H}_{26}$ and the corresponding $\mathrm{E}^{(2)}$ value of this interaction is $0.82 \mathrm{kcal} / \mathrm{mol}$. We thus conclude that the interactions between the $\mathrm{n}\left(\mathrm{N}_{17}\right)$ lone pair of 2-AP and the $\pi^{*}\left(\mathrm{C}_{3}-\right.$ $\left.\mathrm{O}_{8}\right)$ and $\pi *\left(\mathrm{C}_{1}-\mathrm{C}_{2}\right)$ orbital of DDQ play a crucial role in the stabilization of $\mathbf{S} 1$ and the 2-AP/DDQ interaction is further reinforced by the $\mathrm{O}_{8} \ldots \mathrm{H}_{26}$ hydrogen bonding.

The main driving forces for the formation of $\mathbf{S 2}$ and $\mathbf{S 3}$ are the interactions $\operatorname{LP}(\sigma) \mathrm{N}_{21} \rightarrow \mathrm{BD}^{*}(\pi) \mathrm{C}_{6}-$ $\mathrm{O}_{7} / \mathrm{BD}^{*}(\pi) \mathrm{C}_{1}-\mathrm{C}_{2}$, and their $\mathrm{E}^{(2)}$ values are 4.25 , $1.36 \mathrm{kcal} / \mathrm{mol}$ for $\mathbf{S 2}$, and $4.21,1.59 \mathrm{kcal} / \mathrm{mol}$ for S3, respectively. The stabilizations are improved by the intermolecular $\pi-\pi$ interactions between the bond- 
Table 2. The selected local orbital interactions and their $\mathrm{E}^{(2)}$ values of S1-S4 (threshold: $0.5 \mathrm{kcal} / \mathrm{mol}$ ).

\begin{tabular}{|c|c|c|c|}
\hline Conformer & Donor NBO(i) & Acceptor NBO(j) & $\mathrm{E}^{(2)}(\mathrm{kcal} / \mathrm{mol})$ \\
\hline \multirow[t]{5}{*}{ S1 } & $\mathrm{BD}(\pi) \mathrm{C}_{3}-\mathrm{O}_{8}$ & $\mathrm{BD}^{*}(\sigma) \mathrm{N}_{21}-\mathrm{H}_{26}$ & 1.02 \\
\hline & $\mathrm{LP}(\sigma) \mathrm{O}_{8}$ & $\mathrm{BD}^{*}(\sigma) \mathrm{N}_{21}-\mathrm{H}_{26}$ & 0.82 \\
\hline & $\mathrm{BD}(\pi) \mathrm{N}_{17}-\mathrm{C}_{18}$ & $\mathrm{BD}^{*}(\pi) \mathrm{C}_{3}-\mathrm{O}_{8}$ & 0.76 \\
\hline & $\mathrm{LP}(\sigma) \mathrm{N}_{17}$ & $\mathrm{BD}^{*}(\pi) \mathrm{C}_{1}-\mathrm{C}_{2}$ & 3.06 \\
\hline & $\mathrm{LP}(\sigma) \mathrm{N}_{17}$ & $\mathrm{BD}^{*}(\pi) \mathrm{C}_{3}-\mathrm{O}_{8}$ & 5.97 \\
\hline \multirow[t]{4}{*}{ S2 } & $\mathrm{BD}(\pi) \mathrm{N}_{17}-\mathrm{C}_{18}$ & $\mathrm{BD}^{*}(\pi) \mathrm{C}_{1}-\mathrm{C}_{2}$ & 1.31 \\
\hline & $\mathrm{BD}(\pi) \mathrm{N}_{17}-\mathrm{C}_{18}$ & $\mathrm{BD}^{*}(\pi) \mathrm{C}_{3}-\mathrm{O}_{8}$ & 1.11 \\
\hline & $\operatorname{LP}(\sigma) \mathrm{N}_{21}$ & $\mathrm{BD}^{*}(\pi) \mathrm{C}_{1}-\mathrm{C}_{2}$ & 1.36 \\
\hline & $\operatorname{LP}(\sigma) \mathrm{N}_{21}$ & $\mathrm{BD}^{*}(\pi) \mathrm{C}_{6}-\mathrm{O}_{7}$ & 4.25 \\
\hline \multirow[t]{5}{*}{ S3 } & $\mathrm{BD}(\pi) \mathrm{N}_{17}-\mathrm{C}_{18}$ & $\mathrm{BD}^{*}(\pi) \mathrm{C}_{3}-\mathrm{O}_{8}$ & 1.23 \\
\hline & $\mathrm{BD}(\pi) \mathrm{N}_{17}-\mathrm{C}_{18}$ & $\mathrm{BD}^{*}(\pi) \mathrm{C}_{4}-\mathrm{C}_{5}$ & 0.77 \\
\hline & $\mathrm{BD}(\pi) \mathrm{C}_{19}-\mathrm{C}_{20}$ & $\mathrm{BD}^{*}(\pi) \mathrm{C}_{1}-\mathrm{C}_{2}$ & 0.91 \\
\hline & $\mathrm{LP}(\sigma) \mathrm{N}_{21}$ & $\mathrm{BD}^{*}(\pi) \mathrm{C}_{1}-\mathrm{C}_{2}$ & 1.59 \\
\hline & $\operatorname{LP}(\sigma) N_{21}$ & $\mathrm{BD} *(\pi) \mathrm{C}_{6}-\mathrm{O}_{7}$ & 4.21 \\
\hline \multirow[t]{3}{*}{ S4 } & $\mathrm{LP}(\pi) \mathrm{O}_{8}$ & $\mathrm{BD}^{*}(\sigma) \mathrm{N}_{21}-\mathrm{H}_{26}$ & 0.76 \\
\hline & $\mathrm{LP}(\pi) \mathrm{Cl}_{10}$ & $\mathrm{BD}^{*}(\sigma) \mathrm{N}_{21}-\mathrm{H}_{26}$ & 1.09 \\
\hline & $\mathrm{LP}(\sigma) \mathrm{N}_{17}$ & $\mathrm{BD}^{*}(\sigma) \mathrm{C}_{4}-\mathrm{Cl}_{10}$ & 2.95 \\
\hline
\end{tabular}

ing orbital of 2-AP and antibonding orbital of DDQ, and these interactions are characterized by $\mathrm{BD}(\pi) \mathrm{N}_{17}-$ $\mathrm{C}_{18} \rightarrow \mathrm{BD}^{*}(\pi) \mathrm{C}_{1}-\mathrm{C}_{2} / \mathrm{BD}^{*}(\pi) \mathrm{C}_{3}-\mathrm{O}_{8}$ for $\mathbf{S 2}, \mathrm{BD}(\pi)$ $\mathrm{N}_{17}-\mathrm{C}_{18} \rightarrow \mathrm{BD}^{*}(\pi) \mathrm{C}_{3}-\mathrm{O}_{8}$ and $\mathrm{BD}(\pi) \mathrm{C}_{19}-\mathrm{C}_{20} \rightarrow$ $\mathrm{BD}^{*}(\pi) \mathrm{C}_{1}-\mathrm{C}_{2}$ for $\mathbf{S 3}$, respectively. As indicated in Table 2, the main interactions of $\mathbf{S 4}$ turn out to be halogen bonding and hydrogen bonding interactions, which are characterized by $\operatorname{LP}(\sigma) \mathrm{N}_{17} \rightarrow \mathrm{BD}^{*}(\sigma) \mathrm{C}_{4}-\mathrm{Cl}_{10}$ and $\mathrm{LP}(\pi) \mathrm{Cl}_{10} / \mathrm{LP}(\pi) \mathrm{O}_{8} \rightarrow \mathrm{BD}^{*}(\sigma) \mathrm{N}_{21}-\mathrm{H}_{26}$, and the corresponding $\mathrm{E}^{(2)}$ values are $2.95 \mathrm{kcal} / \mathrm{mol}, 0.76 \mathrm{kcal} / \mathrm{mol}$ and $1.09 \mathrm{kcal} / \mathrm{mol}$, respectively.

\subsection{AIM analysis}

It is well known that Bader's topological analysis of charge density $\rho(r)$ could be widely employed to investigate the intermolecular interactions. ${ }^{26-30}$ In this approach, the location of bond critical point (BCP), the value of charge density $\rho(r)$ and its Laplacian charge density $\nabla^{2} \rho(\mathrm{r})$ at the BCP provide vital information of the nature and strength of the interactions in the formation of complexes. The intermolecular interactions between 2-AP and DDQ in S1-S4, identified via AIM analysis are shown in Figure 2. The values of charge densities and their Laplacians at the BCPs are tabulated in Table 3.

As shown in Figure 2 and Table 3, there are three intermolecular BCPs in $\mathbf{S 1}$; one in the path joining $\mathrm{C}_{3}$ and $\mathrm{N}_{17}$ and the other two in the path joining $\mathrm{O}_{8}$ and $\mathrm{H}_{26}$, and $\mathrm{C}_{1}$ and $\mathrm{H}_{23}$. At the $\mathrm{BCP}$ between $\mathrm{C}_{3}$ and $\mathrm{N}_{17}$, the charge density $\rho(\mathrm{r})$ and its Laplacian $\nabla^{2} \rho($ r) are 0.0193 a.u. and 0.0646 a.u., respectively. The $\mathrm{O}_{8} \cdots \mathrm{H}_{26}$ and $\mathrm{C}_{1} \cdots \mathrm{H}_{23}$ BCPs hint the existence of weak hydrogen bonds between $\mathrm{O}_{8}$ and $\mathrm{H}_{26}$ (or $\mathrm{C}_{1}$ and $\mathrm{H}_{23}$ ). The presence of four BCPs in the stacking region of $\mathbf{S 2}$ and $\mathbf{S 3}$ reveal that there exist intermolecular interactions between 2-AP and DDQ. As indicated in Table 3, The charge densities $\rho(r)$ at the four BCPs range from 0.0070 a.u. to 0.0150 a.u., and their Laplacian $\nabla^{2} \rho(r)$ are in the range of 0.0230 a.u. to 0.0480 a.u.. As displayed in Figure 2, all the topographical features in $\mathbf{S 2}$ and $\mathbf{S 3}$ are similar. The intermolecular dispersion plays a considerable role in the stabilization of the two complexes in addition to the electrostatic interaction. The BCP located between $\mathrm{N}_{17}$ and $\mathrm{Cl}_{10}$ of $\mathbf{S} 4$ indicates the existence of halogen bonding interaction between $\mathrm{N}_{17}$ and $\mathrm{Cl}_{10}$. The $\mathrm{O}_{8} \ldots \mathrm{H}_{26}$ and $\mathrm{Cl}_{10} \ldots \mathrm{H}_{26}$ BCPs reveal that there exist two different hydrogen bonding interaction in S4. As shown in Table 3, all the charge densities at the BCPs are relatively small, and their Laplacian are positive; it indicates that the intermolecular interactions are typical close-shell interactions. All the AIM analysis results are in agreement with those of NBO analysis.

\subsection{UV-Vis absorption spectra and TDDFT calculation}

Analytical grade 2-AP and DDQ were used without further purification. The solutions of 2-AP and DDQ were prepared in chloroform, and the corresponding concentrations are $7.56 \mathrm{mmol} / \mathrm{L}$ and $0.73 \mathrm{mmol} / \mathrm{L}$, respectively. The solutions of 2-AP and DDQ were mixed at the same 


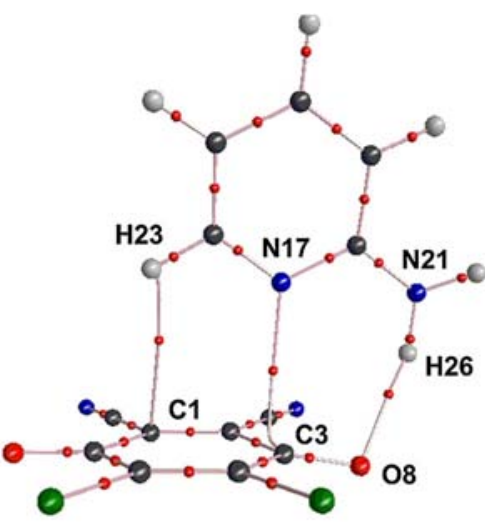

S1

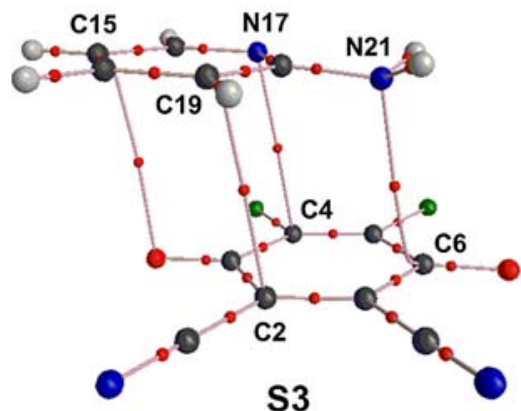

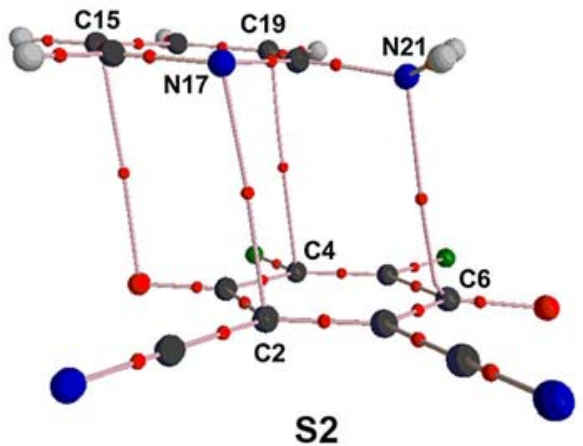

S2

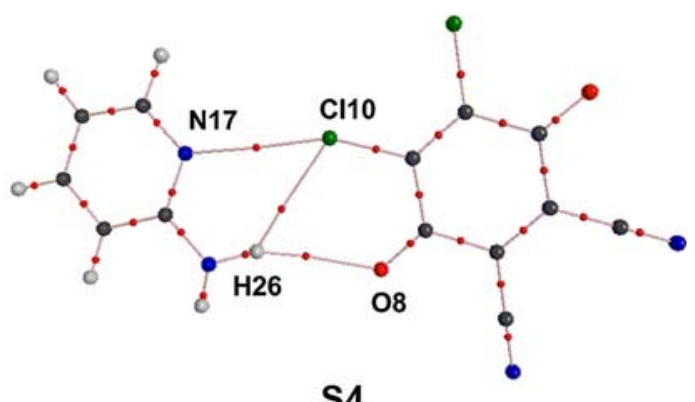

S4

Figure 2. Charge density topography features of S1-S4 (ring critical points (RCPs) and cage critical points (CCPs) are omitted).

Table 3. The charge densities (a.u.) and their Laplacians (a.u.) at the intermolecular BCPs of S1-S4 calculated with M06-2XD3/6-311G(d,p) method.

\begin{tabular}{llll}
\hline Conformer & BCPs & $\rho(\mathrm{r})$ & $\nabla^{2} \rho(\mathrm{r})$ \\
\hline S1 & $\mathrm{C}_{1} \ldots \mathrm{H}_{23}$ & 0.0070 & 0.0211 \\
& $\mathrm{C}_{3} \ldots \mathrm{N}_{17}$ & 0.0193 & 0.0646 \\
\multirow{4}{*}{ S2 } & $\mathrm{O}_{8} \ldots \mathrm{H}_{26}$ & 0.0144 & 0.0731 \\
& $\mathrm{C}_{2} \ldots \mathrm{N}_{17}$ & 0.0121 & 0.0385 \\
& $\mathrm{C}_{4} \ldots \mathrm{C}_{19}$ & 0.0099 & 0.0282 \\
& $\mathrm{C}_{6} \ldots \mathrm{N}_{21}$ & 0.0147 & 0.0473 \\
$\mathbf{5} 3$ & $\mathrm{O}_{8} \ldots \mathrm{C}_{15}$ & 0.0080 & 0.0243 \\
& $\mathrm{C}_{2} \ldots \mathrm{C}_{19}$ & 0.0113 & 0.0322 \\
& $\mathrm{C}_{4} \ldots \mathrm{N}_{17}$ & 0.0103 & 0.0319 \\
& $\mathrm{C}_{6} \ldots \mathrm{N}_{21}$ & 0.0148 & 0.0473 \\
S4 & $\mathrm{O}_{8} \ldots \mathrm{C}_{15}$ & 0.0078 & 0.0233 \\
& $\mathrm{O}_{8} \ldots \mathrm{H}_{26}$ & 0.0077 & 0.0271 \\
& $\mathrm{Cl}_{10} \ldots \mathrm{H}_{26}$ & 0.0085 & 0.0320 \\
& $\mathrm{Cl}_{10} \ldots \mathrm{N}_{17}$ & 0.0152 & 0.0531 \\
& & &
\end{tabular}

volume, and the concentrations of 2-AP and DDQ in the mixture are $3.78 \mathrm{mmol} / \mathrm{L}$ and $0.365 \mathrm{mmol} / \mathrm{L}$, respectively. UV-Vis absorption spectra of the molecules and the mixture were measured after $30 \mathrm{~min}$ using a quartz cell of $0.5 \mathrm{~cm}$ light path, and the spectral slit width

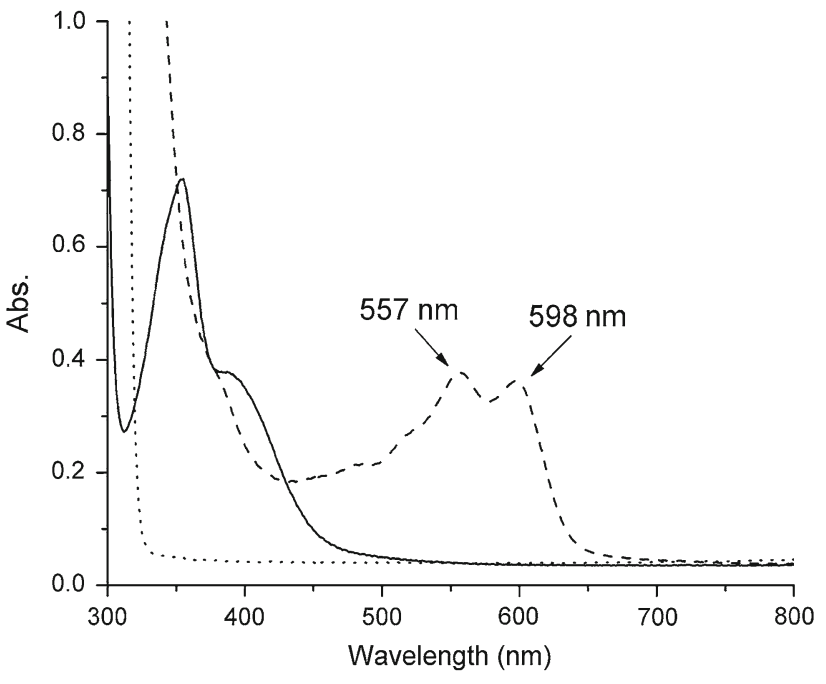

Figure 3. UV-Vis absorption spectra of 2-AP (dot), DDQ (solid) and the 2-AP/DDQ complex (dash) in chloroform

was $2 \mathrm{~nm}$. As displayed in Figure 3, compared with the absorption of the individual molecules, there are two new absorption bands at $557 \mathrm{~nm}$ and $598 \mathrm{~nm}$, which are assigned to the charge transfer absorption of the 2AP/DDQ complex. 

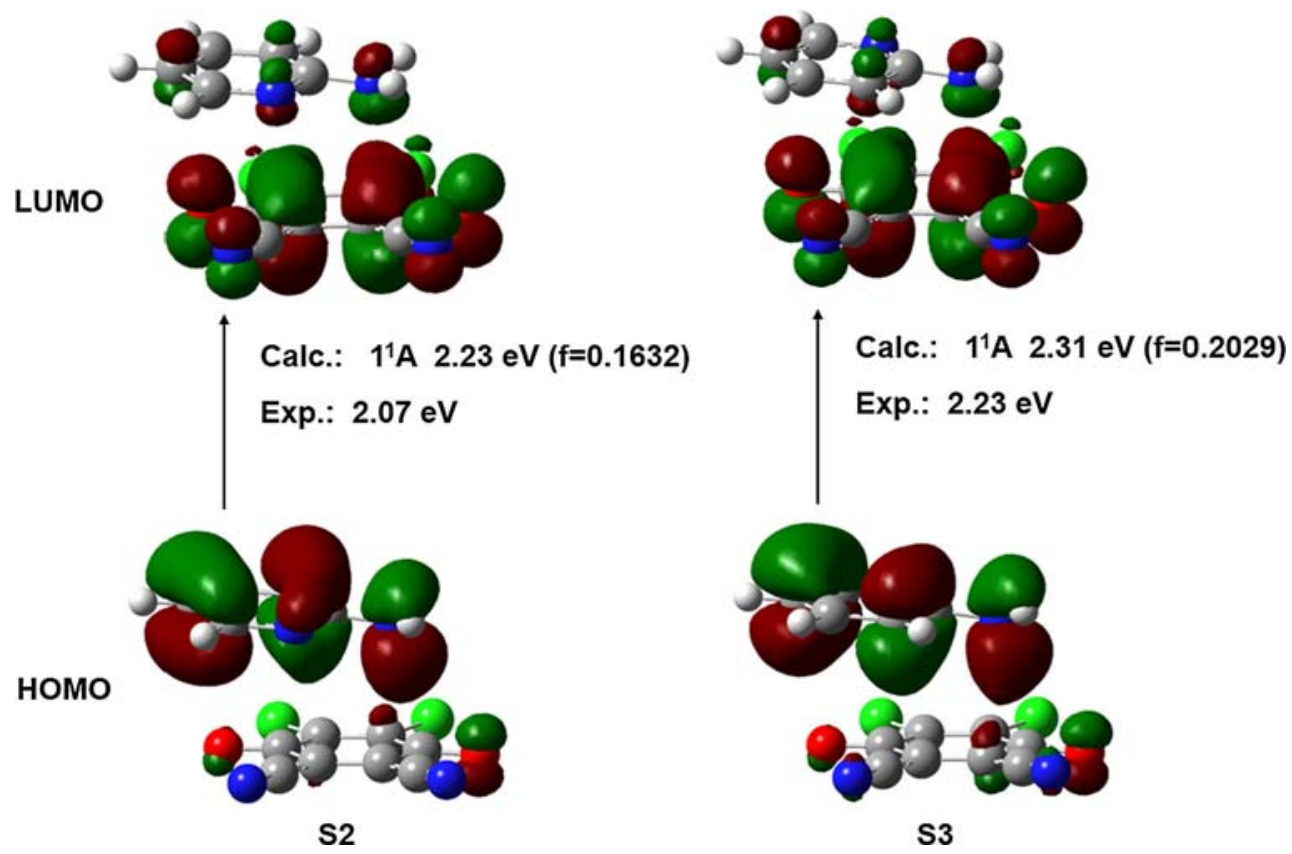

Figure 4. The calculated frontier molecular orbitals, excitation energies $(\Delta \mathrm{E}$ in $\mathrm{eV})$ and oscillator strengths $(f)$ of $\mathbf{S 2}$ (left) and $\mathbf{S 3}$ (right); the experimental data were obtained from the solution spectrum in chloroform.

In order to elucidate the nature of the observed UVVis absorption of 2-AP/DDQ complex, particularly, the two new charge transfer absorption bands, the excitation energies from $S_{0}$ to $S_{30}$ states of the more stable conformers (i.e., $\mathbf{S 2}$ and $\mathbf{S 3}$ ) were calculated using TDDFT with PBE1PBE/6-311G(d,p) methods based on the optimized ground state geometries, because the energy needed for the isomerization of the two conformers is only about $1.00 \mathrm{~kJ} / \mathrm{mol}$. For simplicity, only the first lowest energy transition, namely $S_{0}$ to $S_{1}$ is discussed here. The solvent was chloroform in which the UV-Vis spectroscopy of the complex was performed. The calculated frontier molecular orbitals, excitation energies and oscillator strengths $(f)$ in chloroform are displayed in Figure 4.

As shown in Figure 4, the calculated excitation energies of the first excited singlet states (from $S_{0}$ to $S_{1}$ ) are $2.23 \mathrm{eV}$ and $2.31 \mathrm{eV}$ for $\mathbf{S} 2$ and $\mathbf{S 3}$, respectively, which are closer to the two new absorption bands (2.07 eV and $2.23 \mathrm{eV}$ ) observed experimentally. As a result, it indicates that for the studied system, PBE1PBE method gives better excited state properties in comparison with the experimental data. As shown in Figure 4, The HOMOs and the LUMOs of $\mathbf{S 2}$ and $\mathbf{S 3}$ are almost constructed by the HOMO of 2-AP and the LUMO of $\mathrm{DDQ}$, which are the $2-\mathrm{AP}(\pi) \rightarrow \operatorname{DDQ}\left(\pi^{*}\right)$ charge transfer transitions in character. Hence, we attribute the calculated first excited singlet states of $\mathbf{S 2}$ and $\mathbf{S 3}$ to the two new charge transfer absorption bands observed at $598 \mathrm{~nm}(2.07 \mathrm{eV})$ and $557 \mathrm{~nm}(2.23 \mathrm{eV})$ in Figure 3, respectively.

\section{Conclusions}

In summary, the probable structures and their binding energies of the 2-AP/DDQ complex have been studied using density functional theory. The calculated results located four different stable conformers (denoted as $\mathbf{S 1 - S 4 ) , ~ a n d ~} \mathbf{S 2}$ is more stable than the other conformers. Natural population analysis indicated a net charge transfer from 2-AP to DDQ. Natural bond orbital and atoms-in-molecule analysis revealed that $\mathbf{S 1}$ is formed by the edge(2-AP)-to-face(DDQ) linkage, which is considered in favor of $\sigma-\pi$ and hydrogen binding interactions. S2 and S3 are formed by the face(2-AP)-to-face(DDQ) linkage through $\sigma-\pi$ and $\pi-\pi$ interactions. S4 is constructed by the edge(2AP)-to-edge(DDQ) linkage through halogen bonding and hydrogen bonding interactions. UV-Vis absorption spectra of 2-AP, DDQ and the 2-AP/DDQ complex were recorded in chloroform. Compared with the absorption spectra of 2-AP and DDQ, two new absorption bands at $557 \mathrm{~nm}$ and $598 \mathrm{~nm}$ were observed in 2AP/DDQ complex, which are attributed to the charge transfer absorption of the 2-AP/DDQ complex. The 
electronic excitations of the 2-AP/DDQ complex (S2 and S3) were calculated with TDDFT, which revealed that the $2-\operatorname{AP}(\pi) \rightarrow \operatorname{DDQ}\left(\pi^{*}\right)$ charge transfer transitions in conformers $\mathbf{S} 2$ and $\mathbf{S 3}$ are responsible for the two new charge transfer absorption bands of the 2-AP/DDQ complex.

\section{Acknowledgements}

This project was financially supported by the Department of Education of Zhejiang Province (Y201330088).

\section{References}

1. Horiuchi S, Hasegawa T and Tokura Y 2006 Molecular donor-acceptor compounds as prospective organic electronics materials J. Phys. Soc. Jpn. 75051016

2. Goetz K P, Vermeulen D, Payne M E, Kloc C, McNeil L E and Jurchescu O D 2014 Charge-transfer complexes: New perspectives on an old class of compounds J. Mater. Chem. C 23065

3. Das A and Ghosh S 2014 Supramolecular assemblies by charge-transfer interactions between donor and acceptor chromophores Angew. Chem. Int. Ed. 532038

4. Budzáka Š, Mach P, Juhász G, Medved' M and Kysel' O 2015 Theoretical study of charge transfer complexes between antithyroid thioamides and TCNE: Thermodynamics of the complex formation Comput. Theor. Chem. 1051129

5. Habeeb M M, Al-Attas A S and Al-Raimi D S 2015 Spectroscopic studies and molecular orbital calculations of charge transfer complexation between 3,5dimethylpyrazole with DDQ in acetonitrile Spectrochim. Acta A $\mathbf{1 4 2} 196$

6. Li Z-Y, Wang H-L, He T-J, Liu F-C and Chen D-M 2006 Theoretical study on the structure and UV-visible spectrum of pyridine-chloranil charge-transfer complex $J$. Mol. Struct.-THEOCHEM 77869

7. Al-Ahmary K M, El-Kholy M M, Al-Solmy I A and Habeeb M M 2013 Spectroscopic studies and molecular orbital calculations on the charge transfer reaction between DDQ and 2-aminopyridine Spectrochim. Acta A 110343

8. Bazzi H S, Mostafa A, AlQaradawi S Y and Nour E M 2007 Synthesis and spectroscopic structural investigations of the charge-transfer complexes formed in the reaction of 2,6-diaminopyridine with $\pi$-acceptors TCNE, chloranil, and DDQ J. Mol. Struct. 8421

9. Mostafa A and Bazzi H S 2009 Synthesis and spectroscopic studies of the charge transfer complexes of 2- and 3-aminopyridine Spectrochim. Acta A $\mathbf{7 4} 180$

10. Sobolewskia A L and Domcke W 2003 Ab initio study of the excited-state coupled electron-proton-transfer process in the 2-aminopyridine dimmer Chem. Phys. 294 73

11. Müller A, Talbot F and Leutwyler S 2002 Hydrogen bond vibrations of 2-aminopyridine. 2-pyridone, a WatsonCrick analogue of adenine - uracil J. Am. Chem. Soc. 24 14486
12. Rabenstein B, Ullmann G M and Knapp E W 2000 Electron transfer between the quinones in the photosynthetic reaction center and its coupling to conformational changes Biochemistry 3910487

13. Lieffrig J, Jeannin O, Shin K S, Auban-Senzier P and Fourmigué M 2002 Halogen bonding interactions in DDQ charge transfer salts with iodinated TTFs Crystals 2327

14. Zhang Y-H, Zhou K-G, Xie K-F, Zeng J, Zhang H-L and Peng Y 2010 Tuning the electronic structure and transport properties of graphene by noncovalent functionalization: Effects of organic donor, acceptor and metal atoms Nanotechnology 21065201

15. Hohenstein E G, Chill S T and Sherrill S D 2008 Assessment of the performance of the M05-2X and M06-2X exchange-correlation functionals for noncovalent interactions in biomolecules J. Chem. Theory Comput. 41996

16. Rosokha S V and Loboda E A 2015 Interplay of halogen and $\pi-\pi$ charge-transfer bondings in intermolecular associates of bromo- or iododinitrobenzene with tetramethyl-p-phenylenediamine J. Phys. Chem. A 1193833

17. Karir G, Fatima M and Viswanathan K S 2016 The elusive $\equiv \mathrm{C}-\mathrm{H} \cdots \mathrm{O}$ complex in the hydrogen bonded systems of phenylacetylene: A matrix isolation infrared and Ab Initio study J. Chem. Sci. 1281557

18. Budzák Š, Mach P, Medved'a M and Kysel'a O 2015 Critical analysis of spectral solvent shifts calculated by the contemporary PCM approaches of a representative series of charge-transfer complexes between tetracyanoethylene and methylated benzenes Phys. Chem. Chem. Phys. 1717618

19. Boys S F and Bernardi F 1970 The calculation of small molecular interactions by the differences of separate total energies. Some procedures with reduced errors $\mathrm{Mol}$. Phys. 19553

20. Reed A E, Curtiss L A and Weinhold F 1988 Intermolecular interactions from a natural bond orbital, donor-acceptor viewpoint Chem. Rev. 88899

21. Biegler-KÄonig F, SchÄonbohm J and Bayles D 2002 Update of the AIM 2000-Program for atoms in molecules J. Comput. Chem. 231489

22. Tomasi J, Mennucci B and Cances E 1999 The IEF version of the PCM solvation method: An overview of a new method addressed to study molecular solutes at the QM ab initio level J. Mol. Struct.-THEOCHEM 464211

23. Tomasi J, Mennucci B and Cammi R 2005 Quantum Mechanical Continuum Solvation Models Chem. Rev. 1052999

24. Frisch M J et al. 2009 Gaussian 09, Revision D.01; Gaussian, Inc., Wallingford, CT

25. Bondi A 1964 Van der waals volumes and radii J. Phys. Chem. 68441

26. Misiaszeka T and Czyżnikowska Z 2014 The nature of interactions in nicotinamide crystal J. Mol. Graph. Model. 5173

27. Mohan N and Suresh C H 2014 A molecular electrostatic potential analysis of hydrogen, halogen, and dihydrogen bonds J. Phys. Chem. A 1181697

28. Szatylowicz H, Jezierska A and Sadlej-Sosnowska N 2016 Correlations of NBO energies of individual hydro- 
gen bonds in nucleic acid base pairs with some QTAIM parameters Struct. Chem. 27367

29. Li H-Y, Lu Y-X, Liu Y-T, Zhu X, Liu H-L and Zhu W-L 2012 Interplay between halogen bonds and $\pi-\pi$ stacking interactions: CSD search and theoretical study Phys. Chem. Chem. Phys. 149948
30. Shyam Vinod Kumar P, Raghavendra V and Subramanian V 2016 Bader's theory of atoms in molecules (AIM) and its applications to chemical bonding J. Chem. Sci. 1281527 